\title{
Utilidad de la elastografía cuantitativa por ultrasonografía endoscópica (USE) para el diagnóstico de las lesiones sólidas del páncreas (LSP)
}

\section{Utility of quantitative elastography by Endoscopic Ultrasound (EUS) to diagnose solid pancreatic lesions (SPL)}

\author{
Martín Alonso Gómez-Zuleta, ${ }^{*}$ (1) Óscar Fernando Ruiz-Morales, ${ }^{2}$ (1) Diego Fernando Cano-Rosales. ${ }^{3}$ [C]
}

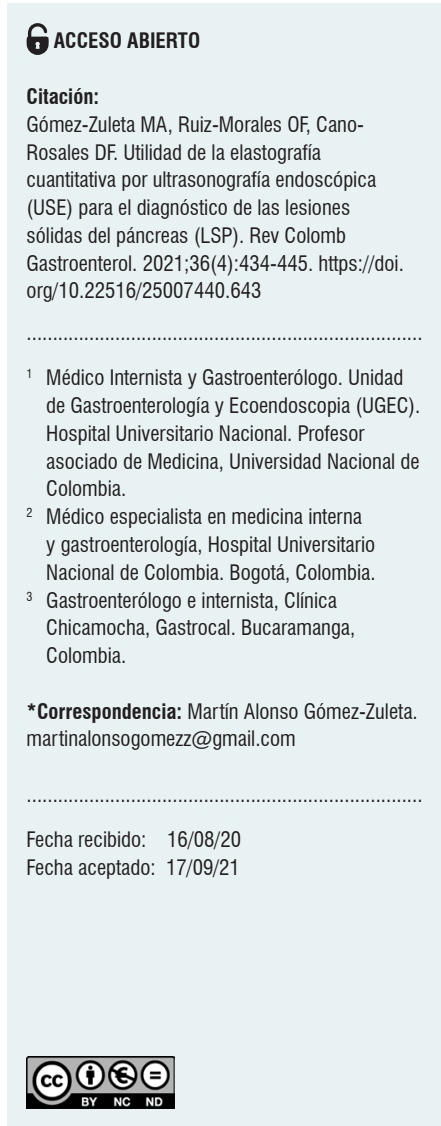

\begin{abstract}
Resumen
Introducción: la punción con aguja fina guiada por ultrasonografía endoscópica (PAF-USE) permite un diagnóstico de las lesiones sólidas del páncreas (LSP) con una sensibilidad de alrededor del $85 \%$ en la literatura mundial y aún más baja en nuestro medio, por lo cual se requiere explorar nuevos accesorios (agujas) 0 técnicas tales como la elastografía, que mejoren esta sensibilidad. Esta última permite la cuantificación de la rigidez del tejido con altos grados de precisión y desde 2001 se ha aplicado al diagnóstico de tumores sólidos de diversos órganos como mama y tiroides, músculo, entre otros; y desde 2006 se ha empleado para las LSP y ha demostrado su utilidad como complemento a las herramientas diagnósticas disponibles, ya que mejora la precisión de la biopsia por PAF-USE al seleccionar el área más sospechosa para ser puncionada y también guía el manejo clínico cuando la PAF-USE es negativa o no concluyente. Objetivo: evaluar el rendimiento diagnóstico de la elastografía cuantitativa de strain ratio (SR) obtenida por ecoendoscopia en las LSP teniendo como patrón de oro el diagnóstico citopatológico. Métodos: 71 pacientes (rango de edad: 35-89, media: 62,2 años); de estos, 35 mujeres fueron sometidas a USE para la evaluación de LSP. El diseño del estudio fue de corte transversal, prospectivo y de un solo centro. La USE se realizó con un ecoendoscopio Pentax lineal y un procesador Hitachi-Noblus. La lesión (área A) y un área de referencia B se seleccionaron para calcular la relación de deformación (B/A, SR expresada en \%). Se tomó como punto de corte SR para definir las lesiones malignas (duras) $S R>22$ teniendo en cuenta la evidencia actualmente disponible; estos resultados se compararon con la citopatología de las muestras obtenidas por punción guiada por USE. Después de la aplicación de criterios de exclusión, se realiza el análisis estadístico de 56 pacientes y se considera el valor $p<0,05$. Se calculó la sensibilidad, especificidad, valor predictivo positivo (VPP), valor predictivo negativo (VPN) y precisión diagnóstica comparando la elastografía SR con los diagnósticos finales por citopatología. Resultados: la elastografía cuantitativa SR (\%) permite detectar las LSP malignas con sensibilidad del 94,6 $\%$ (intervalo de confianza [IC] del $95 \%$ : 85,4 \%-98,2 \%), especificidad del 89,3\% (IC 95 \%: 78,5 \%-95,0 \%), VPP del 89,8 \% (IC $95 \%$ : 79,5 \%-95,3\%); VPN del 94,3\% (IC $95 \%$ : 84,6 \%-98,1\%) y exactitud del 92,0\% (IC $95 \%$ : 85,4 \%-95,7\%). Conclusión: la elastografía cuantitativa SR por USE en LSP es un complemento útil que mejora la precisión de la PAF-USE al seleccionar el área más sospechosa para ser puncionada y guiar el manejo clínico cuando la PAF-USE es negativa o no concluyente, ya que tiene una alta sensibilidad y especificad en el diagnóstico de las LSP malignas.
\end{abstract}

\section{Palabras clave}

Elastografía, ultrasonido endoscópico, lesión sólida pancreática, elasticidad, páncreas.

\section{INTRODUCCIÓN}

La inserción a la práctica clínica de la ultrasonografía endoscópica (USE) ha permitido un avance importante en el trata- miento de una amplia gama de patologías, cambiando significativamente el diagnóstico o el manejo de estas en un $25 \%$ a un $50 \%$ de los $\operatorname{casos}^{(1,2)}$. En el páncreas, la USE permite obtener imágenes de alta resolución del parénquima y del 


\begin{abstract}
Introduction: Endoscopic ultrasonography with fine-needle aspiration allows performing a diagnosis of solid pancreatic lesions with an approximate $85 \%$ sensitivity, as referenced in specialized literature, and even lower sensitivity as per local research. To yield better sensitivity and to improve the results, it is required to examine new elements (needles) and techniques like elastography. Elastography helps in the quantification of tissue stiffness with a high level of accuracy. Since 2001, elastography has been applied in diagnosing solid forms of cancer (tumors) that affect organs like breasts, the thyroid, and some muscles. This method which has been used to diagnose solid pancreatic lesions (SPL) since 2006 has proved to be useful as a complementary method to the existing diagnostic techniques. It improves the accuracy of the endoscopic ultrasound-guided fine-needle aspiration biopsy (EUS-FNA) by selecting the more suspicious area to be punctured, and it also guides the clinical treatment after getting a negative EUS-FNA or a non-conclusive result. Objective: To evaluate the diagnostical performance of the strain ratio (SR) quantitative elastography by ecoendoscopy in solid pancreatic lesions, considering the cytopathologic diagnostic as the gold standard. Methods: 71 patients (age range: $35-89$ years old, mean: 62.2 years old); out of those 71 patients, The EUS to diagnose SPL, was performed on 35 women. This was a single-center, prospective cross-sectional study design. The EUS was performed with a Pentax linear endoscope and a Hitachi-Noblus ultrasound. The lesion (area A) and a reference area $B$ were selected to calculate the deformation ratio (B/A, SR expressed as a percentage). SR > 22 was selected as a cut-off point to determine the malignant lesions (solid lesions), considering the evidence currently available. The results were compared with their cytopathology interpretation once that the EUS was performed. After the exclusion criteria was applied, a statistical analysis of 56 patients was performed, considering $p<0,05$. The sensitivity, the specificity, the positive predictive value (PPV), the negative predictive value (NPV) and the diagnostic accuracy, were calculated, comparing the elastography SR with the final diagnostics with the cytopathology interpretation. Results: Quantitative elastography SR (\%) allows to detect the malignant SPL with sensitivity $94.6 \%$ (95\% confidence interval [Cl]: $85.4 \%-98.2 \%)$, specificity of $89.3 \%(\mathrm{Cl}$ 95\%: 78,5\%-95,0 \%), PPV of 89.8\% (CI 95\%: 79,5\%-95,3\%); NPV of $94.3 \%$ (IC 95 \%: 84,6 \%-98,1 \%) and an accuracy of $92.0 \%$ (Cl $95 \%: 85,4 \%-95,7 \%$ ). Conclusion: SR quantitative elastography by Endoscopic Ultrasound, EUS is a suitable complement method that improves the EUS-FNA accuracy, by selecting the most suspicious area to be punctured, and it also guides clinical treatment after getting a negative EUS-FNA or a non-conclusive result, due to its high sensitivity and specificity levels to diagnose malignant SPL.
\end{abstract}

\title{
Keywords
}

Elastography, Endoscopic ultrasound, Solid pancreatic lesion, Elasticity, Pancreas.

conducto, por lo que se considera un método para la estadificación y diagnóstico de diferentes entidades, ya sean consideradas como enfermedades pancreáticas benignas o malignas; y se destaca su papel en el diagnóstico diferencial de las lesiones sólidas del páncreas (LSP), las cuales representan un grupo heterogéneo de entidades que pueden ser clasificadas como neoplásicas o no neoplásicas, y el adenocarcinoma ductal es el tumor maligno más frecuentemente detectado, hasta en el $90 \%$ de todas las neoplasias malignas pancreáticas $^{(3,4)}$.

El cáncer de páncreas es una causa significativa de mortalidad con una tasa de supervivencia a 5 años menor del $5 \%$ y alcanzan el $20 \%$ en pacientes seleccionados (tumores no invasivos llevados a resección quirúrgica $)^{(5)}$. Sin embargo y a pesar de su utilidad demostrada, la USE no siempre puede determinar un diagnóstico preciso utilizando solo imágenes, por lo que la obtención de tejido del páncreas mediante la toma de células por aspiración con aguja fina guiada por PAF-USE (punción con aguja fina guiada por ultrasonografía endoscópica) permite un diagnóstico definitivo con un buen rendimiento ${ }^{(1,6,7)}$, con una sensibilidad aproximada del $85 \%$ al $90 \%$, con falsos negativos del $15 \%$ y con especificidades que se acercan al $100 \%(7,8)$.

A pesar de este rendimiento, existen diferentes factores que lo afectan su de forma sustancial, ya que se trata de un procedimiento exigente desde el punto de vista técnico que puede requerir múltiples punciones con el fin de obtener una muestra suficiente para hacer un adecuado diagnóstico ${ }^{(9)}$. Además, existen otros factores que también afectan este rendimiento, dentro de los cuales están la variabilidad interobservador, la falta de estandarización de la técnica, la experiencia del ecoendoscopista, la posición del equipo, el momento del día, el calibre de aguja utilizado, la técnica, las características de las lesiones, la cantidad de pases de la aguja, la presencia del citólogo en la sala, la presencia de pancreatitis crónica, entre otros ${ }^{(10-17)}$. Por estas razones han surgido nuevos métodos no invasivos que permiten una caracterización más precisa de estas lesiones, mientras que la toma de PAF-USE se limita a los pacientes con lesiones altamente sospechosas de malignidad. Una de estas técnicas se llama elastografía ${ }^{(1,18)}$, la cual es un procedimiento no invasivo que emergió con base en conceptos descritos desde 1988, como los de deformabilidad tisular y elasti- 
cidad de un tumor sólido ${ }^{(19)}$; después, en 1991 las mediciones de elasticidad del tejido se realizaron mediante la evaluación de un módulo de elasticidad después de aplicar una presión utilizando el término de elastografía por primera vez ${ }^{(2,21)}$. Esto llevó al desarrollo de la combinación de imágenes en tiempo real de ecografía en modo B en $2001^{(21,22)}$. Desde entonces, la elastografía se ha aplicado al diagnóstico de tumores sólidos de diversos órganos, como mama, tiroides, ganglios linfáticos e hígado; pero hasta 2006 se informó por primera vez el uso de la elastografía para $\operatorname{LSP}^{(21,23,24)}$.

Desde el punto de vista técnico, la elastografía se basa en el fundamento de que la presión ejercida a una lesión diana por una sonda de ecoendoscopia crea tensión (strain), que difiere según la dureza o suavidad del tejido, lo que permite diferenciar los tejidos considerados benignos como suaves y los tejidos malignos como duros. La tensión generada a los tejidos se representa a través de diferentes colores basados en su elasticidad (rojo es el tejido más blando y azul es el tejido más duro) ${ }^{(21,25)}$. Existen dos sistemas diferentes de elastografía disponibles: el primero se basa en la evaluación cualitativa del patrón obtenido del estudio elastográfico (elastografía cualitativa: se observa el color de la lesión; si es azul homogéneo, sugiere malignidad), mientras que el segundo se basa en una cuantificación de la rigidez realizada por el software (elastografía cuantitativa) ${ }^{(1,25)}$. La ventaja más importante de la elastografía por USE es que puede proporcionar al endoscopista datos en tiempo real durante la evaluación diagnóstica, lo que puede afectar el manejo de los pacientes al valorar la naturaleza de la lesión y dirigirse con mayor precisión cuando se va a tomar la muestra de citología por el PAF-USE, sin la necesidad de un segundo tiempo endoscópico o estudios diagnósticos adicionales; aunque es importante mencionar que la elastografía por USE aún no se considera una modalidad que pueda reemplazar la biopsia. Puede ser un complemento útil, debido a que mejora la precisión de la biopsia por PAF-USE al seleccionar el área más sospechosa para ser puncionada y, además, puede ser útil para guiar el manejo clínico adicional cuando la PAF-USE es negativa o no es concluyente ${ }^{(18,25)}$.

En Colombia no se tienen estudios clínicos que muestren el rendimiento diagnóstico de la elastografía por USE en las LSP, por lo que, en este estudio, evaluamos cuál es el rendimiento diagnóstico de la elastografía cuantitativa o el strain ratio (SR) en la diferenciación de las LSP.

\section{MATERIALES Y MÉTODOS}

\section{Objetivo primario}

Evaluar el rendimiento diagnóstico de la elastografía cuantitativa SR obtenida por ecoendoscopia en las LSP teniendo como patrón el diagnóstico histopatológico.

\section{Diseño del estudio}

Estudio de corte transversal, prospectivo, entre enero de 2017 y enero de 2018 en una unidad de referencia en gastroenterología y ecoendoscopia, el estudio fue aprobado por el comité ético de la unidad y se realizó de acuerdo con la Declaración de Helsinki y sus enmiendas, y las pautas de Buenas Prácticas Clínicas ${ }^{(26)}$.

\section{Pacientes}

Se realizó un total de 682 ecoendoscopias en este período, de las cuales 71 (10,56\%) fueron pacientes referidos para punción de LSP; este grupo de pacientes fue seleccionado teniendo en cuenta los siguientes criterios de inclusión: pacientes mayores de 18 años de edad con diagnóstico de LSP, informe ecoendoscópico que incluía realización de elastografía cuantitativa SR y toma de punción guiada por ecoendoscopia con aguja fina, resultado de citotopatología de muestras de punción conclusivo y firma de consentimiento informado. Los criterios de exclusión fueron: pacientes con funcionalidad disminuida mayor a 4 en la escala de ECOG (Eastern Cooperative Oncology Group) (Tabla 1) ${ }^{(27)}$, pacientes con riesgo de hemorragia (índice internacional normalizado [INR]) $>1,5$ o con recuento de plaquetas $<50$ $000 / \mathrm{mm}^{2}$, pacientes que consumen 2 o más agentes antiagregantes plaquetarios, pacientes con masa pancreática que no se logra detectar por USE, pacientes en los que no se logra la punción por variantes anatómicas (interposición de grandes vasos, anatomía quirúrgica alterada), mujer embarazada, pacientes menores de 18 años y pacientes que no autorizaban la inclusión de sus datos al estudio. Después de la aplicación de los criterios se incluyeron 56 pacientes para el análisis.

\section{Análisis estadístico}

La recolección de los datos de la población incluida fue realizada a través de tablas de datos virtuales de Google Drive realizadas simultáneamente con el procedimiento por un médico internista fellow de gastroenterología previamente entrenado para diligenciar el formulario virtual; estos datos fueron corregidos e ingresados en el software SPSS (versión 12.0; SPSS Inc.). Los datos de SR fueron probados usando análisis de varianza unidireccional y se consideró un valor $p<0,05$ para indicar significancia estadística. Los datos se presentan como medias, rangos, porcentajes e intervalos de confianza (IC) del $95 \%$, según corresponda; la sensibilidad, la especificidad, el valor predictivo positivo (VPP), el valor predictivo negativo (VPN) y la precisión se calcularon comparando los diagnósticos realizados por elastografía SR y los diagnósticos finales dados por citopatología, la cual se obtuvo mediante un aspirado tomado por PAF-USE. 
0 Totalmente activo, capaz de llevar a cabo todas las actividades de la vida diaria, previo a la enfermedad sin restricción.

1 El paciente presenta síntomas que le impiden realizar trabajos arduos, aunque se desempeña normalmente en sus actividades cotidianas y en trabajos ligeros.

El paciente solo permanece en la cama durante las horas de sueño nocturno.

2 El paciente no es capaz de desempeñar ningún trabajo, se encuentra con síntomas que le obligan a permanecer en la cama durante varias horas al día, además de las de la noche, pero que no superan el $50 \%$ del día.

El individuo satisface la mayoría de sus necesidades personales solo.

3 El paciente necesita estar en cama más de la mitad del día por la presencia de síntomas. Necesita ayuda para la mayoría de las actividades de la vida diaria; por ejemplo, para vestirse.

4 El paciente permanece en cama el $100 \%$ del día y necesita ayuda para todas las actividades de la vida diaria, como en la higiene corporal, la movilización en la cama e incluso la alimentación.

5 Muerto.

Tomada de la referencia ${ }^{(27)}$.

\section{Técnica}

La USE en todos los pacientes fue realizada siguiendo los indicadores de calidad de la American Society for Gastrointestinal Endoscopy y American College of Gastroenterology $y^{(28)}$, se llevaron a cabo en la sala de gastroenterología de una unidad de referencia para la realización de procedimientos endoscópicos diagnósticos, bajo sedación guiada por anestesiólogo, con una combinación de propofol más remifentanilo, titulada según las características de cada paciente. Todos los procedimientos fueron realizados con un ecoendoscopio marca Pentax lineal (EG3870UTK; Pentax, Tokio, Japón) combinado con un sistema de ultrasonido portátil Doppler color modelo Noblus (Hitachi Aloka Medical, Tokio, Japón), que incluye el módulo de elastografía y fueron realizados por un endoscopista experimentado en procedimientos intervencionistas, con más de 1500 ecoendoscopias con punción realizadas. Se utilizaron agujas de aspiración para ecoendoscopia número 22 Gauge (Boston Scientific).

Se utilizó la técnica de succión húmeda para la toma de biopsias con un total de 5 pases y 4 movimientos dentro de la lesión, según las recomendaciones descritas en la literatura, dado su mayor rendimiento diagnóstico $(85,2 \%)$ frente a la técnica seca $(71 \%)$ en nuestra población (resultados en proceso de publicación). En esta técnica, antes de puncionar la lesión se retira el estilete (aguja calibre 22) y se prelava con $5 \mathrm{~mL}$ de solución salina para reemplazar la columna de aire con líquido. Una jeringa de $10 \mathrm{~mL}$ se prellena con $3 \mathrm{~mL}$ de solución salina y se utiliza para aspi- rar posteriormente al realizar la punción de la lesión. Una vez la aguja está dentro de la lesión se mueve 3 veces de un lado para otro, esta maniobra se repite 4 veces (pases) para un total de 12 movimientos. Al retirar la aguja, el aspirado se libera en una lámina aplicando aire ${ }^{(29-31)}$. Además del mayor rendimiento diagnóstico, un metaanálisis demostró que había una menor tasa de sangrado cuando se utiliza esta técnica ${ }^{(32)}$. Las muestras se fijaron en alcohol etílico y fueron enviadas para estudio citopatológico por un especialista en patología.

Para la evaluación de la elastografía, se marcaron diferentes valores de elasticidad con varios colores que dieron como resultado diferentes patrones elastográficos, que se mostraron superpuestos en imágenes de USE convencionales en modo B, siguiendo las recomendaciones técnicas disponibles en la literatura publicada hasta el momento ${ }^{(25,33)}$. Como resultante, la representación de colores de los tejidos duros, intermedios y blandos son azul, verde/amarillo y rojo, respectivamente. Para la elastografía, el ecoendoscopio lineal se maniobró hacia la luz gastrointestinal administrando la tensión necesaria para generar una imagen óptima en modo B en 7,5 MHz. La región de interés (ROI) para la evaluación elastográfica se eligió manualmente para abarcar toda el área objetivo de la LSP (la mayor parte cuando las dimensiones de la lesión no lo permitían), así como los tejidos circundantes. Se requirió una imagen estable de al menos 5 segundos de duración para el análisis cuantitativo y la definición final del patrón.

Se eligieron dos áreas diferentes (A y B) dentro de la región de interés para el análisis elastográfico cuantitativo. 
El área A incluye toda la lesión (cuando fue posible), mientras que el área B incluye el área de referencia grasa peripancreática fuera del tumor (tejido blando graso) ${ }^{(25,33,34)}$. El SR fue calculado por el software del procesador expresado en porcentaje teniendo en cuenta las áreas de interés (ROI) realizando un cálculo de la relación $\mathrm{B} / \mathrm{A}^{(25,35)}$. Para limitar el sesgo de selección de las áreas A y B, sus valores de elasticidad se estimaron tres veces en todos los pacientes, el valor medio de las tres medidas de SR se consideró el resultado final del análisis. Se tomó como punto de corte de SR para definir la presencia de lesiones malignas (duras) los valores $>22$, teniendo en cuenta los datos publicados sobre el diagnóstico diferencial de LSP, en especial el punto de corte recientemente identificado en un estudio realizado en 398 pacientes usando los valores de SR para detectar específicamente cáncer pancreático, que fue específicamente de $21,80 \pm 12,23^{(36)}$, punto de corte similar al encontrado por Itokawa y colaboradores; y de 39,08 $\pm 20,54$ también para cáncer pancreático ${ }^{(37)}$, y se estimaron las recomendaciones sobre la técnica publicadas ${ }^{(25)}$.

\section{RESULTADOS}

Un total de 682 ecoendoscopias fueron realizadas entre enero de 2017 y enero de 2018, de las cuales 71 (10,56\%), con un rango de edad de 25-89 años (media de 62,28 años), fueron diagnosticadas con LSP; el tamaño de las lesiones evaluadas tenía un rango de $15-60 \mathrm{~mm}$ con una media de 29,34 mm, el rango de SR (\%) obtenido fue de 12-189 (media de 51,15). Por una parte, de estos pacientes, 35 (49,3\%) fueron de sexo femenino, con rango de edad de 25-89 años (media: 63,32 años), el tamaño de la lesión en este grupo fue de $15-55 \mathrm{~mm}$ (media: 30,8041 ) y el rango SR (\%) en este grupo fue de 13-189 (media: 50,436). Por otra parte, $36(50,7 \%)$ pacientes fueron de sexo masculino, con un rango de edad de 29-87 años (media: 61,24), el tamaño de lesiones tuvo un rango en milímetros de 15-60 $\mathrm{mm}$ (media: 27,97 mm), el rango de SR (\%) en este grupo fue de 12-140 (media: 51,86) (Tabla 2).

Al $100 \%$ de los pacientes les fue realizada la punción, pero en 11 pacientes (6 mujeres y 5 hombres [15,5\%]) las muestras no fueron suficientes para llegar a una conclusión diagnóstica por parte de patología, $2(2,8 \%)$ de los pacientes de sexo femenino no dieron su autorización para el ingreso al estudio y con 2 de los pacientes $(2,8 \%)$ de sexo femenino y masculino no se logra la comunicación para conocer el resultado de estudio histopatológico. El análisis estadístico se realizó en 56 pacientes, 24 de sexo femenino $(42,8 \%)$ y 32 de sexo masculino (58,2\%) (Figura 1), los diagnósticos ecoendoscópicos y su frecuencia y los diagnósticos encontrados posteriormente en el estudio histopatológico se resumen en la Tabla 2.

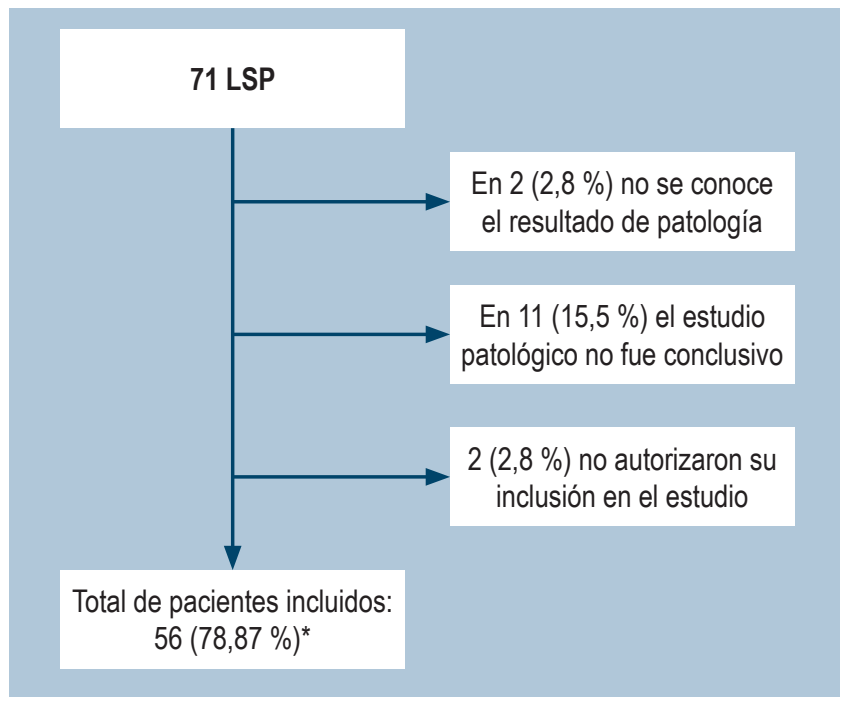

Figura 1. Diagrama de flujo de inclusión de pacientes en el estudio. *56 pacientes fueron incluidos en el análisis estadístico después de la aplicación de criterios de inclusión y exclusión propuestos en el diseño metodológico del estudio.

$\mathrm{Al}$ hacer el análisis estadístico se encontró que la elastografía cuantitativa por SR (\%) permite detectar las masas pancreáticas malignas con una sensibilidad del 94,6\% (IC $95 \%: 85,4 \%-98,2 \%$ ), especificidad del 89,3 \% (IC $95 \%$ : 78,5 \%-95,0 \%), valor predictivo positivo (VPP) del 89,8\% (IC $95 \%: 79,5 \%-95,3 \%$ ); valor predictivo negativo (VPN) del 94,3 \% (IC $95 \%: 84,6 \%-98,1 \%$ ), exactitud del 92,0 \% (IC $95 \%: 85,4 \%-95,7 \%$ ).

\section{DISCUSIÓN}

La prevalencia de LSP no es tan clara. Strang y colaboradores informaron una prevalencia de $0,6 \%$ de masas pancreáticas en donantes de riñón potencialmente $\operatorname{sanos}^{(38)}$; una prevalencia similar de 0,49\% entre 2941 pacientes sometidos a tomografía por emisión de positrones con 18F-fluorodesoxiglucosa (FDG-PET) por causas no relacionadas $^{(39)}$. En Japón, de 39785 exploraciones con FDGPET realizadas para la detección de cáncer, la prevalencia de neoplasia pancreática fue inferior al $0,001 \%{ }^{(40)}$; una cifra más estrechamente relacionada con SEER (Survelliance, Epidemiology and End Results program, Estados Unidos), que muestra una incidencia general de $0,73 \%$ para 2013, la cual ha aumentado ${ }^{(41)}$.

El diagnóstico diferencial de las LSP es amplio ya que representan un grupo heterogéneo de entidades que pueden ser clasificadas como neoplásicas y no neoplásicas. Las lesiones neoplásicas (también llamadas malignas) son las más comunes e incluyen el adenocarcinoma, tumores neuroendocrinos, tumor pseudopapilar sólido, pancreatoblastoma, linfoma, metástasis y neoplasias misceláneas más $\operatorname{raras}^{(42,43)}$. 
Tabla 2. Características de los pacientes

\begin{tabular}{|c|c|c|c|c|c|c|c|c|}
\hline Sexo & $\mathrm{n}$ & $\%$ & $\begin{array}{l}\text { Rango de } \\
\text { edad (años) }\end{array}$ & Media & $\begin{array}{l}\text { Tamaño de lesiones } \\
\text { (rango en mm) }\end{array}$ & Media & SR (\%) & Media \\
\hline Femenino & 35 & 49,3 & $25-89$ & 63,32671062 & $15-55$ & 30,8041 & $13-189$ & 50,436 \\
\hline Masculino & 36 & 50,7 & $29-87$ & 61,24544003 & $15-60$ & 27,9732 & $12-140$ & 51.867 \\
\hline Total & 71 & 100 & $25-89$ & 62,27738159 & $15-60$ & 29,336 & $12-189$ & 51,1516 \\
\hline \multicolumn{9}{|l|}{ Diagnóstico ecoendoscópico } \\
\hline - Cáncer en la cabeza del páncreas & 51 & 71,8 & & & & & & \\
\hline - Cistoadenocarcinoma & 1 & 1,4 & & & & & & \\
\hline - Lesión de cabeza y cuerpo del páncreas & 2 & 2,8 & & & & & & \\
\hline - Lesión del cuerpo del páncreas & 4 & 5,6 & & & & & & \\
\hline - Lesión focal en la cabeza del páncreas & 6 & 8,4 & & & & & & \\
\hline - Pancreatitis crónica & 2 & 2,8 & & & & & & \\
\hline - Tumor de Franz & 2 & 2,8 & & & & & & \\
\hline - Cáncer en la cola del páncreas & 2 & 2,8 & & & & & & \\
\hline - Lesión en el cuello de páncreas & 1 & 1,4 & & & & & & \\
\hline - Total & 71 & 100 & & & & & & \\
\hline \multicolumn{9}{|l|}{ Diagnóstico histopatológico } \\
\hline - Adenocarcinoma & 48 & 67,6 & & & & & & \\
\hline - No diagnóstico & 15 & 21,1 & & & & & & \\
\hline - Pancreatitis crónica & 4 & 5,6 & & & & & & \\
\hline - Tumor sólido pseudopapilar & 2 & 2,8 & & & & & & \\
\hline - Lesión mesenquimal con atipia & 1 & 1,4 & & & & & & \\
\hline - Neoplasia papilar oncocítica & 1 & 1,4 & & & & & & \\
\hline - Total & 71 & 100 & & & & & & \\
\hline - No cumplen con los criterios para el análisis & 15 & 21,1 & & & & & & \\
\hline $\begin{array}{l}\text { - Total de pacientes incluidos en el análisis } \\
\text { estadístico }\end{array}$ & 56 & 78,9 & & & & & & \\
\hline
\end{tabular}

Como ya se mencionó, el adenocarcinoma ductal es el tumor maligno más frecuente del páncreasy representa cerca del $90 \%$ de todas las neoplasias malignas pancreáticas ${ }^{(3-5,44,45)}$. La American Cancer Society estima que 48960 casos de cáncer de páncreas se desarrollaron en 2015 en los Estados Unidos y la mayoría de los pacientes (40 560) morirán de la enfermedad $^{(46)}$. La tasa de supervivencia a 5 años es menor del $5 \%$, alcanzando su punto más alto, de un $20 \%$, en pacientes seleccionados con tumores no invasivos a quienes les fue realizada una resección quirúrgica ${ }^{(47)}$.
La sintomatología en general no ocurre hasta que la enfermedad esté avanzada y frecuentemente se presenta con ictericia obstructiva, dolor abdominal, anorexia, pérdida de peso, pancreatitis aguda, diabetes de nueva aparición o mal controlada, o esteatorrea. En la exploración física se pueden encontrar hallazgos como ictericia, desgaste muscular, lesiones cutáneas, adenopatías palpables, hepatomegalia o masas palpables. En ocasiones, estas lesiones se encuentran de manera incidental en las pruebas de imágenes abdominales de vigilancia, durante la evaluación del dolor abdo- 
minal o en pacientes que presentan anormalidades en las pruebas de rutina o de diagnóstico en el perfil hepático. Especialmente el aumento de los niveles de bilirrubina y fosfatasa alcalina puede conducir a un diagnóstico de colestasis debido a la obstrucción biliar cuando la LSP se localiza en la cabeza del páncreas; sin embargo, la gran mayoría de pacientes cursa sin ningún síntoma, por lo que el principal objetivo es detectarlo en estadios tempranos. Actualmente, el ultrasonido, la tomografía axial computarizada (TAC) y las imágenes por resonancia magnética (RM) son el pilar en la evaluación del $80 \%-85 \%$ de las $\operatorname{LSP}^{(48,49)}$.

El reto del diagnóstico preoperatorio de las LSP persiste a pesar del avance en las imágenes. La PAF-USE se considera el método de elección para detectar y diagnosticar estas lesiones ${ }^{(6,44)}$. El rendimiento diagnóstico es altamente sensible y específico ${ }^{(50)}$, aunque existen múltiples factores que afectan su rendimiento, lo que ha impulsado el desarrollo de nuevos métodos diagnósticos basados en el avance tecnológico, dentro de los cuales está la elastografía, la cual se ha utilizado con gran éxito para evaluar otros órganos distintos al páncreas como mama, tiroides, próstata, cuello uterino, hígado, músculos y otros ${ }^{(51-54)}$.

La elastografía se basa en el conocimiento de que diferentes patologías generan procesos como la fibrosis y la inflamación, lo cual aumenta la rigidez de los tejidos. Las pulsaciones vasculares fisiológicas y los movimientos respiratorios proporcionan las vibraciones y compresiones necesarias que toma el software para el estudio, es así como la elastografía se construye de manera tal que utiliza principalmente la información de la onda de pulso aórtico ${ }^{(1,55,56)}$. La elastografía se puede realizar en tiempo real utilizando un equipo de ecoendoscopia convencional conectado a un procesador con el software específico instalado ${ }^{(1,54-56)}$. Se considera una técnica fácil de aplicar cuando se realiza una endosonografía, no requiere de preparación adicional, ni cambios en la posición del paciente, ni en el procedimiento anestésico.

Existen dos diferentes generaciones en la elastografía por USE, la primera generación permitía solo evaluación cualitativa. Giovannini y colaboradores ${ }^{(53)}$ publicaron el primer estudio sobre elastografía guiada por USE en LSP utilizando la evaluación cualitativa. Considerando las lesiones azules (duras) como malignas, se reportó una sensibilidad y especificidad para malignidad del $100 \%$ y $67 \%$, respectivamente. Con base en este estudio, se definió un sistema de puntuación (Tabla 3) que permitía hacer una clasificación menos subjetiva de la elastografía $^{(57)}$. En 2009 Giovannini y colaboradores, en un estudio multicéntrico con 121 casos, informaron que la elastografía por ecoendoscopia de USE en la diferenciación entre masas pancreáticas benignas y malignas alcanzó una sensibilidad 92,3\%, con una especificidad de $80,0 \%$, un VPP de $93,3 \%$ y un VPN de $77,4 \%$, con una precisión general de $89,2 \%$. Publicado en ese mismo año, Iglesias-García y colaboradores ${ }^{(58)}$ evaluaron a 130 pacientes con masas pancreáticas sólidas y 20 controles, definieron 4 patrones diferentes de la elastografía y reportaron una sensibilidad del $100 \%$, especificidad del $85,5 \%$, VPP de 90,7 \%, VPN del $100 \%$ y una precisión de $94,0 \%$. Todos los sujetos fueron evaluados por 2 endosonografistas que hicieron la misma interpretación en 121 de 130 casos y 20 de 20 controles, con un índice kappa de 0,772 como evaluador de concordancia. Para 2015, Soares J-B y colaboradores evaluaron la concordancia interobservador y concluyeron que la elastografía guiada por USE es reproducible en la evaluación de LSP, incluso entre ecoendoscopistas sin experiencia o con experiencia limitada ${ }^{(59)}$. Sin embargo y a pesar de los resultados descritos, se trata de una técnica subjetiva y dependiente del operador, que tiene como principal problema la reproducibilidad de la imagen dentro del procedimiento, por lo que la investigación siguió avanzando ${ }^{(60)}$.

Tabla 3. Clasificación de patrones elastográficos para USE

\begin{tabular}{lcc|}
\hline \multicolumn{1}{c}{ Color y patrón } & Rigidez & Malignidad \\
\hline Homogéneo, predomina el verde & Suave & No \\
\hline Heterogéneo, predomina el verde & Intermedio & No \\
Heterogéneo, predomina el azul & Duro & $\mathrm{Si}$ \\
Homogéneo, predomina el azul & Duro & $\mathrm{Si}$ \\
$\begin{array}{l}\text { Heterogéneo, verde y azul sin color } \\
\text { predominante }\end{array}$ & $\begin{array}{c}\text { Intermedio } \\
\text { duro }\end{array}$ & Indeterminada \\
\hline
\end{tabular}

Tomada de la referencia ${ }^{(57)}$.

Actualmente, la elastografía por USE de segunda generación, además de la evaluación cualitativa, también permite una estimación cuantitativa de la rigidez del tejido con 2 enfoques diferentes: relación de deformación (SR) y el histograma de deformación. El más ampliamente difundido y con mayor cantidad de estudios es el SR, por lo que fue tomado como criterio base del estudio; el SR compara la tensión entre el área objetivo A y otras áreas de referencia $\mathrm{B}$, para proporcionar datos cualitativos más objetivos ${ }^{(1,37)}$. Diferentes estudios utilizan SR para diagnosticar diferencialmente el carcinoma pancreático ${ }^{(53,61-67)}$. Todos ellos tienen variantes en la toma de las áreas de interés a ROI, algunos de ellos toman el tejido pancreático sano, tejido peripancreático y la grasa mesentérica, y otros toman la pared duodenal como referencia para obtener el SR. En nuestro estudio se tomó la grasa peripancreática como comparador de las lesiones (ROI B), teniendo en cuenta que se trata de un tejido blando que se puede evaluar 
fácilmente en la misma imagen o corte ecográfico dada la cercanía anatómica de estas estructuras y que es el patrón utilizado en las lesiones de mama, con las que inicialmente fue descrita esta técnica ${ }^{(68-70)}$.

El rendimiento diagnóstico de la elastografía cualitativa SR se ha evaluado a través del tiempo en diferentes metaanálisis. En 2012 la elastografía tuvo una sensibilidad del $95 \%$ (IC $95 \%$ : $93 \%-96 \%$ ) y una especificidad del $69 \%$ (IC $95 \%$ : 63-75\%) ${ }^{(71)}$; en 2013 se evaluaron 13 estudios con 1042 casos y una sensibilidad del $95 \%$ (IC $95 \%$ : $94 \%$ $97 \%$ ), una especificidad de $67 \%$ (IC $95 \%$ : $61 \%-73 \%$ ), y un Odds ratio (OR) de 42,28 (IC $95 \%: 26,90-66,46)^{(72)}$. También en 2013 otro metaanálisis incluyó 7 estudios con 752 pacientes y encontró que la sensibilidad de la elastografía por USE para el diagnóstico diferencial de masas pancreáticas sólidas fue del $97 \%$ (IC $95 \%$ : 0,95-0,98) y la especificidad fue del 76 \% (IC $95 \%$ : 0,69-0,82). El área bajo la curva (ROC) fue de 0,9529. La razón de probabilidad positiva combinada fue de 3,71 (IC $95 \%: 2,72-5,07$ ) y la razón de probabilidad negativa fue de 0,05 (IC $95 \%$ : $0,02-0,13)^{(73)}$. Otro metaanálisis publicado en este mismo año incluyó 10 estudios con un total de 781 pacientes y reportó que la sensibilidad fue de $92 \%$ (IC $95 \%$ : 0,890,95), la especificidad del $76 \%$ (IC $95 \%: 0,67-0,83$ ), razón de verosimilitud positiva (LR [+]) del 2,84 (IC $95 \%: 2,05$ 3,93), razón de verosimilitud negativo (LR [-]) de 0,12 (IC $95 \%$ : 0,08-0,19) y OR diagnóstico de 24,69 (IC $95 \%$, $12,81-47,59)^{(74)}$. Sin embargo, todos estos estudios muestran una heterogeneidad significativa dada por la variabilidad entre ensayos clínicos controlados tomados para su realización, principalmente en la escogencia de diferentes áreas de ROI (el área de referencia blanda B); otro factor a tener en cuenta es la distancia del área de referencia desde el ecoendoscopio, que también impacta significativamente las mediciones de $\mathrm{SR}^{(75)}$, y de diferentes puntos de corte de SR, sumados a la subjetividad de la elastografía cualitativa.

Mas recientemente, en 2018 un nuevo metaanálisis que incluyó un total de 19 estudios que reunieron 1687 pacientes a los cuales se les realizó la elastografía cuantitativa mostraron una sensibilidad del $95 \%$ (IC del $95 \%$ : 0,93-0,97), especificidad del $61 \%$ (IC $95 \%: 0,56$ a 0,66), LR (+) de 2,64 (IC $95 \%: 1,82-3,82$ ) y LR (-) de 0,10 (IC $95 \%$ : 0,06$0,16)^{(76)}$. En nuestro estudio se encontró una sensibilidad del 94,6 \% (IC $95 \%$ : 85,4\%-98,2 \%), lo cual corresponde a las cifras de sensibilidad reportadas en los metaanálisis; sin embargo, la especificidad fue del 89,3 \% (IC 95 \%: 78,5\%$95,0 \%)$, lo cual es un poco más alta de lo reportado en la literatura; nosotros explicamos este hallazgo debido a que nuestros pacientes tenían lesiones más avanzadas y más grandes (en promedio, $30 \mathrm{~mm}$ ), lo cual aumenta significativamente la posibilidad de malignidad ( $86,9 \%-93,2 \%)$ $(77,78)$. La edad de los pacientes y la metodología permitieron la disminución de factores de confusión previamente identificados en la literatura, como la toma de grasa peripancreática como comparador (tejido suave, ROI B), la toma del promedio de 3 diferentes SR en el mismo paciente, el uso de un punto de corte estándar previamente evaluado ( $>22$ ), la experiencia del endosonografista y la utilización de equipos con más tecnología. Asimismo, la disminución de factores de confusión también se logró con el seguimiento de las recomendaciones sobre los principios técnicos de la elastografía tisular en tiempo real que fueron descritos recientemente en detalle por la Federación europea de Sociedades de Ultrasonido en Medicina y Biología (EFSUMB) y la Federación Mundial de Ultrasonido en Medicina y Biología (WFUMB). En resumen, estos parámetros son el uso del transductor apropiado y la selección adecuada de la frecuencia, cantidad de cuadros por segundo, línea de densidad, velocidad de palpación y amplitud, filtros de ruido (ultrasonográfico), persistencia, rango dinámico de elasticidad y otros parámetros de calidad ( $\mathrm{p}$. ej., visualización del gráfico de deformación $)^{(70,79-81)}$. Por su parte, el VPP de 89,8 \% (IC 95 \%: 79,5 \%-95,3 \%), VPN de $94,3 \%$ (IC $95 \%: 84,6 \%-98,1 \%$ ) y exactitud del 92,0 \% (IC $95 \%$ : 85,4\%-95,7\%) son resultados congruentes con los encontrados en un estudio recientemente publicado retrospectivo de 116 pacientes (97 con lesiones malignas y 19 con lesiones benignas), con una mediana de edad de 55,9 años; se usó un punto de corte de SR de 7,75 con una especificidad del 99,9\%, sensibilidad del 90,7 \%, VPP del $99,9 \%$, VPN del $67,9 \%$ y precisión del $92,2 \%{ }^{(82)}$.

\section{CONCLUSIÓN}

El diagnóstico diferencial de las LSP sigue siendo uno de los desafíos diagnósticos más difíciles en la práctica clínica. La PAF-USE es el mejor método para diagnosticar tumores sólidos pancreáticos debido a su alta sensibilidad y especificidad cuando se combina con la elastografía cuantitativa. Sin embargo, la elastografía por USE aún no se considera una modalidad que pueda reemplazar la biopsia. Pero cabe resaltar que es un complemento útil, ya que mejora la precisión de la biopsia por PAF-USE al seleccionar el área más sospechosa para ser puncionada. Adicionalmente, es útil para guiar el manejo clínico adicional, cuando la PAF-USE es negativa o no es concluyente, dado que, como muestra este trabajo, es altamente sensible y específica en el diagnóstico de los tumores malignos del páncreas. 


\section{REFERENCIAS}

1. Iglesias-García J, Lariño-Noia J, Domínguez-Muñoz JE. New Imaging Techniques: Endoscopic UltrasoundGuided Elastography. Gastrointest Endosc Clin N Am. 2017;27(4):551-567.

https://doi.org/10.1016/j.giec.2017.06.001

2. Luthra AK, Evans JA. Review of current and evolving clinical indications for endoscopic ultrasound. World J Gastrointest Endosc. 2016;8(3):157-64. https://doi.org/10.4253/wjge.v8.i3.157

3. Kleeff J, Korc M, Apte M, La Vecchia C, Johnson CD, Biankin AV, Neale RE, Tempero M, Tuveson DA, Hruban RH, Neoptolemos JP. Pancreatic cancer. Nat Rev Dis Primers. 2016;2:16022. https://doi.org/10.1038/nrdp.2016.22

4. Bray F, Ferlay J, Soerjomataram I, Siegel RL, Torre LA, Jemal A. Global cancer statistics 2018: GLOBOCAN estimates of incidence and mortality worldwide for 36 cancers in 185 countries. CA Cancer J Clin. 2018;68(6):394-424. https://doi.org/10.3322/caac.21492

5. Xiao AY, Tan ML, Wu LM, Asrani VM, Windsor JA, Yadav D, Petrov MS. Global incidence and mortality of pancreatic diseases: a systematic review, meta-analysis, and metaregression of population-based cohort studies. Lancet Gastroenterol Hepatol. 2016;1(1):45-55. https://doi.org/10.1016/S2468-1253(16)30004-8

6. Wang W, Shpaner A, Krishna SG, Ross WA, Bhutani MS, Tamm EP, Raju GS, Xiao L, Wolff RA, Fleming JB, Lee $\mathrm{JH}$. Use of EUS-FNA in diagnosing pancreatic neoplasm without a definitive mass on CT. Gastrointest Endosc. 2013;78(1):73-80 https://doi.org/10.1016/j.gie.2013.01.040

7. Hewitt MJ, McPhail MJ, Possamai L, Dhar A, Vlavianos P, Monahan KJ. EUS-guided FNA for diagnosis of solid pancreatic neoplasms: a meta-analysis. Gastrointest Endosc. 2012;75(2):319-31. https://doi.org/10.1016/j.gie.2011.08.049

8. Bhatia V, Varadarajulu S. Endoscopic ultrasonographyguided tissue acquisition: How to achieve excellence. Dig Endosc. 2017;29(4):417-430. https://doi.org/10.1111/den.12823

9. Polkowski M, Larghi A, Weynand B, Boustière C, Giovannini M, Pujol B, Dumonceau JM; European Society of Gastrointestinal Endoscopy (ESGE). Learning, techniques, and complications of endoscopic ultrasound (EUS)guided sampling in gastroenterology: European Society of Gastrointestinal Endoscopy (ESGE) Technical Guideline. Endoscopy. 2012;44(2):190-206. https://doi.org/10.1055/s-0031-1291543

10. Korenblit J, Tholey DM, Tolin J, Loren D, Kowalski T, Adler DG, Davolos J, Siddiqui AA. Effect of the time of day and queue position in the endoscopic schedule on the performance characteristics of endoscopic ultrasound-guided fine-needle aspiration for diagnosing pancreatic malignancies. Endosc Ultrasound. 2016;5(2):78-84. https://doi.org/10.4103/2303-9027.180470
11. Ramesh J, Bang JY, Hebert-Magee S, Trevino J, Eltoum I, Frost A, Hasan MK, Logue A, Hawes R, Varadarajulu S. Randomized Trial Comparing the Flexible 19G and 25G Needles for Endoscopic Ultrasound-Guided Fine Needle Aspiration of Solid Pancreatic Mass Lesions. Pancreas. 2015;44(1):128-33. https://doi.org/10.1097/MPA.0000000000000217

12. Madhoun MF, Wani SB, Rastogi A, Early D, Gaddam S, Tierney WM, Maple JT. The diagnostic accuracy of 22-gauge and 25-gauge needles in endoscopic ultrasoundguided fine needle aspiration of solid pancreatic lesions: a meta-analysis. Endoscopy. 2013;45(2):86-92. https://doi.org/10.1055/s-0032-1325992

13. Kamata K, Kitano M, Yasukawa S, Kudo M, Chiba Y, Ogura T, Higuchi K, Fukutake N, Ashida R, Yamasaki T, Nebiki H, Hirose S, Hoki N, Asada M, Yazumi S, Takaoka M, Okazaki K, Matsuda F, Okabe Y, Yanagisawa A. Histologic diagnosis of pancreatic masses using 25-gauge endoscopic ultrasound needles with and without a core trap: a multicenter randomized trial. Endoscopy. 2016;48(7):632-8. https://doi.org/10.1055/s-0042-106294

14. Nakai $Y$, Isayama $H$, Chang KJ, Yamamoto N, Hamada T, Uchino R, Mizuno S, Miyabayashi K, Yamamoto K, Kawakubo K, Kogure H, Sasaki T, Hirano K, Tanaka M, Tada M, Fukayama M, Koike K. Slow pull versus suction in endoscopic ultrasound-guided fine-needle aspiration of pancreatic solid masses. Dig Dis Sci. 2014;59(7):1578-85. https://doi.org/10.1007/s10620-013-3019-9

15. Bang JY, Magee SH, Ramesh J, Trevino JM, Varadarajulu S. Randomized trial comparing fanning with standard technique for endoscopic ultrasound-guided fine-needle aspiration of solid pancreatic mass lesions. Endoscopy. 2013;45(6):445-50. https://doi.org/10.1055/s-0032-1326268

16. Suzuki R, Irisawa A, Bhutani MS, Hikichi T, Takagi T, Sato A, Sato M, Ikeda T, Watanabe K, Nakamura J, Tasaki K, Obara K, Ohira H. Prospective evaluation of the optimal number of 25-gauge needle passes for endoscopic ultrasound-guided fine-needle aspiration biopsy of solid pancreatic lesions in the absence of an onsite cytopathologist. Dig Endosc. 2012;24(6):452-6. https://doi.org/10.1111/j.1443-1661.2012.01311.x

17. Hébert-Magee S, Bae S, Varadarajulu S, Ramesh J, Frost AR, Eloubeidi MA, Eltoum IA. The presence of a cytopathologist increases the diagnostic accuracy of endoscopic ultrasound-guided fine needle aspiration cytology for pancreatic adenocarcinoma: a meta-analysis. Cytopathology. 2013;24(3):159-71. https://doi.org/10.1111/cyt.12071

18. Cui XW, Chang JM, Kan QC, Chiorean L, Ignee A, Dietrich CF. Endoscopic ultrasound elastography: Current status and future perspectives. World J Gastroenterol. 2015;21(47):13212-24. https://doi.org/10.3748/wjg.v21.i47.13212 
19. Ueno E, Tohno E, Soeda S, Asaoka Y, Itoh K, Bamber JC, Blaszçzyk M, Davey J, Mckinna JA. Dynamic tests in realtime breast echography. Ultrasound Med Biol. 1988;14 Suppl 1:53-7. https://doi.org/10.1016/0301-5629(88)90047-6

20. Ophir J, Céspedes I, Ponnekanti H, Yazdi Y, Li X. Elastography: a quantitative method for imaging the elasticity of biological tissues. Ultrason Imaging. $1991 ; 13(2): 111-34$. https://doi.org/10.1177/016173469101300201

21. Chantarojanasiri T, Kongkam P. Endoscopic ultrasound elastography for solid pancreatic lesions. World J Gastrointest Endosc. 2017;9(10):506-513. https://doi.org/10.4253/wjge.v9.i10.506

22. Shiina T, Nitta N, Ueno E, Bamber JC. Real time tissue elasticity imaging using the combined autocorrelation method. J Med Ultrason (2001). 2002;29(3):119-28. https://doi.org/10.1007/BF02481234

23. Giovannini M, Hookey LC, Bories E, Pesenti C, Monges G, Delpero JR. Endoscopic ultrasound elastography: the first step towards virtual biopsy? Preliminary results in 49 patients. Endoscopy. 2006;38(4):344-8. https://doi.org/10.1055/s-2006-925158

24. Gennisson JL, Deffieux T, Fink M, Tanter M. Ultrasound elastography: principles and techniques. Diagn Interv Imaging. 2013;94(5):487-95. https://doi.org/10.1016/j.diii.2013.01.022

25. Costache MI, Dumitrescu D, Săftoiu A. Technique of qualitative and semiquantitative EUS elastography in pancreatic examination. Endosc Ultrasound. 2017;6(Suppl 3):S111-S114. https://doi.org/10.4103/eus.eus 7517

26. Declaración de Helsinki de la AMM. Principios éticos para las investigaciones médicas en seres humanos [internet]. AMM [consultado el 10 de agosto de 2019]. Disponible en: www.wma.net/es/policies-post/declaracion-de-helsinki-de-la-amm-principios-eticos-para-las-investigacionesmedicas-en-seres-humanos

27. Pérez Cruz PE, Acevedo F. Escalas de estado funcional (o performance status) en cáncer. Gastroenterol Latinoam. 2014;25(3):219-226.

28. Wani S, Wallace MB, Cohen J, Pike IM, Adler DG, Kochman ML, Lieb JG 2nd, Park WG, Rizk MK, Sawhney MS, Shaheen NJ, Tokar JL. Quality indicators for EUS. Am J Gastroenterol. 2015;110(1):102-13. https://doi.org/10.1038/ajg.2014.387

29. Villa NA, Berzosa M, Wallace MB, Raijman I. Endoscopic ultrasound-guided fine needle aspiration: The wet suction technique. Endosc Ultrasound. 2016;5(1):17-20. https://doi.org/10.4103/2303-9027.175877

30. Polkowski M, Jenssen C, Kaye P, Carrara S, Deprez P, Gines A, Fernández-Esparrach G, Eisendrath P, Aithal GP, Arcidiacono P, Barthet M, Bastos P, Fornelli A, Napoleon B, Iglesias-Garcia J, Seicean A, Larghi A, Hassan C, van Hooft JE, Dumonceau JM. Technical aspects of endoscopic ultrasound (EUS)-guided sampling in gastroenterology: European Society of Gastrointestinal Endoscopy
(ESGE) Technical Guideline - March 2017. Endoscopy. 2017;49(10):989-1006.

https://doi.org/10.1055/s-0043-119219

31. Attam R, Arain MA, Bloechl SJ, Trikudanathan G, Munigala S, Bakman Y, Singh M, Wallace T, Henderson JB, Catalano MF, Guda NM. "Wet suction technique (WEST)": a novel way to enhance the quality of EUS-FNA aspirate. Results of a prospective, single-blind, randomized, controlled trial using a 22-gauge needle for EUS-FNA of solid lesions. Gastrointest Endosc. 2015;81(6):1401-7. https://doi.org/10.1016/j.gie.2014.11.023

32. Lee JK, Choi JH, Lee KH, Kim KM, Shin JU, Lee JK, Lee $\mathrm{KT}$, Jang KT. A prospective, comparative trial to optimize sampling techniques in EUS-guided FNA of solid pancreatic masses. Gastrointest Endosc. 2013;77(5):745-51. https://doi.org/10.1016/j.gie.2012.12.009

33. Dietrich CF, Bibby E, Jenssen C, Saftoiu A, Iglesias-Garcia J, Havre RF. EUS elastography: How to do it? Endosc Ultrasound. 2018;7(1):20-28. https://doi.org/10.4103/eus.eus_49_17

34. Dietrich CF, Săftoiu A, Jenssen C. Real time elastography endoscopic ultrasound (RTE-EUS), a comprehensive review. Eur J Radiol. 2014;83(3):405-14. https://doi.org/10.1016/j.ejrad.2013.03.023

35. Kamata K, Kitano M, Omoto S, Kadosaka K, Miyata T, Minaga K, Yamao K, Imai H, Kudo M. New endoscopic ultrasonography techniques for pancreaticobiliary diseases. Ultrasonography. 2016;35(3):169-79. https://doi.org/10.14366/usg.15042

36. Kim SY, Cho JH, Kim YJ, Kim EJ, Park JY, Jeon TJ, Kim YS. Diagnostic efficacy of quantitative endoscopic ultrasound elastography for differentiating pancreatic disease. J Gastroenterol Hepatol. 2017;32(5):1115-1122. https://doi.org/10.1111/jgh.13649

37. Itokawa $\mathrm{F}$, Itoi $\mathrm{T}$, Sofuni $\mathrm{A}$, Kurihara $\mathrm{T}$, Tsuchiya $\mathrm{T}$, Ishii K, Tsuji S, Ikeuchi N, Umeda J, Tanaka R, Yokoyama N, Moriyasu F, Kasuya K, Nagao T, Kamisawa T, Tsuchida A. EUS elastography combined with the strain ratio of tissue elasticity for diagnosis of solid pancreatic masses. J Gastroenterol. 2011;46(6):843-53. https://doi.org/10.1007/s00535-011-0399-5

38. Strang AM, Lockhart ME, Kenney PJ, Amling CL, Urban DA, El-Galley R, Burns JR, Colli JL, Hammontree LN, Kolettis PN. Computerized tomographic angiography for renal donor evaluation leads to a higher exclusion rate. J Urol. 2007;177(5):1826-9. https://doi.org/10.1016/j.juro.2007.01.007

39. Pitts A, Nissen NN, Waxman A, Yu R. Unsuspected fluorodeoxyglucose positron emission tomography (FDG-PET)positive pancreatic lesions: prevalence and significance. Pancreas. 2013;42(7):1191-3. https://doi.org/10.1097/MPA.0b013e318287d06e

40. Weckesser M, Schober O. Is whole-body FDG-PET valuable for health screening? Against. Eur J Nucl Med Mol Imaging. 2005;32(3):342-3. https://doi.org/10.1007/s00259-005-1775-2 
41. Gordon-Dseagu VL, Devesa SS, Goggins M, StolzenbergSolomon R. Pancreatic cancer incidence trends: evidence from the Surveillance, Epidemiology and End Results (SEER) population-based data. Int J Epidemiol. 2018;47(2):427-439. https://doi.org/10.1093/ije/dyx232

42. Zárate $X$, Williams N, Herrera MF. Pancreatic incidentalomas. Best Pract Res Clin Endocrinol Metab. 2012;26(1):97-103. https://doi.org/10.1016/j.beem.2011.06.005

43. Low G, Panu A, Millo N, Leen E. Multimodality imaging of neoplastic and nonneoplastic solid lesions of the pancreas. Radiographics. 2011;31(4):993-1015. https://doi.org/10.1148/rg.314105731

44. Kamisawa T, Wood LD, Itoi T, Takaori K. Pancreatic cancer. Lancet. 2016;388(10039):73-85. https://doi.org/10.1016/S0140-6736(16)00141-0

45. Ferlay J, Soerjomataram I, Dikshit R, Eser S, Mathers C, Rebelo M, Parkin DM, Forman D, Bray F. Cancer incidence and mortality worldwide: sources, methods and major patterns in GLOBOCAN 2012. Int J Cancer. 2015;136(5):E359-86. https://doi.org/10.1002/ijc.29210

46. ASGE Standards of Practice Committee, Eloubeidi MA, Decker GA, Chandrasekhara V, Chathadi KV, Early DS, Evans JA, Fanelli RD, Fisher DA, Foley K, Hwang JH, Jue TL, Lightdale JR, Pasha SF, Saltzman JR, Sharaf R, Shergill AK, Cash BD, DeWitt JM. The role of endoscopy in the evaluation and management of patients with solid pancreatic neoplasia. Gastrointest Endosc. 2016;83(1):17-28. https://doi.org/10.1016/j.gie.2015.09.009

47. Liles JS, Katz MH. Pancreaticoduodenectomy with vascular resection for pancreatic head adenocarcinoma. Expert Rev Anticancer Ther. 2014;14(8):919-29. https://doi.org/10.1586/14737140.2014.919860

48. Hanada K, Okazaki A, Hirano N, Izumi Y, Teraoka Y, Ikemoto J, Kanemitsu K, Hino F, Fukuda T, Yonehara S. Diagnostic strategies for early pancreatic cancer. J Gastroenterol. 2015;50(2):147-54. https://doi.org/10.1007/s00535-014-1026-z

49. Scialpi M, Reginelli A, D’Andrea A, Gravante S, Falcone G, Baccari P, Manganaro L, Palumbo B, Cappabianca S. Pancreatic tumors imaging: An update. Int J Surg. 2016;28 Suppl 1:S142-55. https://doi.org/10.1016/j.ijsu.2015.12.053

50. Bang JY, Hebert-Magee S, Navaneethan U, Hasan MK, Hawes R, Varadarajulu S. EUS-guided fine needle biopsy of pancreatic masses can yield true histology. Gut. 2018;67(12):2081-2084. https://doi.org/10.1136/gutjnl-2017-315154

51. Meng FS, Zhang ZH, Ji F. New endoscopic ultrasound techniques for digestive tract diseases: A comprehensive review. World J Gastroenterol. 2015;21(16):4809-16. https://doi.org/10.3748/wjg.v21.i16.4809

52. Hirooka Y, Itoh A, Hashimoto S, Kawashima H, Hara K, Kanamori A, Uchida H, Goto J, Ishikawa S, Ohmiya N, Niwa Y, Goto H. Utility of EUS: Elastography in the Diagnosis of
Pancreatic Diseases. Gastroenterology. 2005;61(5):AB282. https://doi.org/10.1016/S0016-5107(05)01447-1.

53. Okasha H, Elkholy S, El-Sayed R, Wifi MN, El-Nady M, El-Nabawi W, El-Dayem WA, Radwan MI, Farag A, El-Sherif Y, Al-Gemeie E, Salman A, El-Sherbiny M, El-Mazny A, Mahdy RE. Real time endoscopic ultrasound elastography and strain ratio in the diagnosis of solid pancreatic lesions. World J Gastroenterol. 2017;23(32):5962-5968. https://doi.org/10.3748/wjg.v23.i32.5962

54. Arcidiacono PG. Endoscopic ultrasound elastography. Gastroenterol Hepatol (N Y). 2012;8(1):48-67.

55. Sigrist RMS, Liau J, Kaffas AE, Chammas MC, Willmann JK. Ultrasound Elastography: Review of Techniques and Clinical Applications. Theranostics. 2017;7(5):1303-1329. https://doi.org/10.7150/thno.18650

56. Săftoiu, A., Gheonea, D. I., Cârțână, T., \&amp; Streba, C. Advanced endoscopic ultrasound imaging: contrast-enhanced endoscopic ultrasound (low MI, high MI), including $3 \mathrm{D}$ techniques in Pancreatic imaging. Video Journal and Encyclopedia of GI Endoscopy, 2013, 1, 534-536.

57. Janssen J, Schlörer E, Greiner L. EUS elastography of the pancreas: feasibility and pattern description of the normal pancreas, chronic pancreatitis, and focal pancreatic lesions. Gastrointest Endosc. 2007;65(7):971-8. https://doi.org/10.1016/j.gie.2006.12.057

58. Iglesias-Garcia J, Larino-Noia J, Abdulkader I, Forteza J, Dominguez-Munoz JE. EUS elastography for the characterization of solid pancreatic masses. Gastrointest Endosc. 2009;70(6):1101-8. https://doi.org/10.1016/j.gie.2009.05.011

59. Soares JB, Iglesias-Garcia J, Goncalves B, Lindkvist B, Lariño-Noia J, Bastos P, Caetano AC, Ferreira A, PimentelNunes P, Lopes L, Moutinho-Ribeiro P, DominguezMuñoz JE. Interobserver agreement of EUS elastography in the evaluation of solid pancreatic lesions. Endosc Ultrasound. 2015;4(3):244-9. https://doi.org/10.4103/2303-9027.163016

60. Giovannini M. What is the place of pancreatic endoscopic ultrasound elastography in 2018? Endoscopy. 2018;50(11):1051-1052. https://doi.org/10.1055/a-0637-8840

61. Kongkam P, Lakananurak N, Navicharern P, Chantarojanasiri T, Aye K, Ridtitid W, Kritisin K, Angsuwatcharakon P, Aniwan S, Pittayanon R, Sampatanukul P, Treeprasertsuk S, Kullavanijaya P, Rerknimitr R. Combination of EUS-FNA and elastography (strain ratio) to exclude malignant solid pancreatic lesions: A prospective single-blinded study. J Gastroenterol Hepatol. 2015;30(11):1683-9. https://doi.org/10.1111/jgh.13067

62. Dietrich CF, Sahai AV, D’Onofrio M, Will U, Arcidiacono PG, Petrone MC, Hocke M, Braden B, Burmester E, Möller K, Săftoiu A, Ignee A, Cui XW, Iordache S, Potthoff A, Iglesias-Garcia J, Fusaroli P, Dong Y, Jenssen C. Differential diagnosis of small solid pancreatic lesions. Gastrointest Endosc. 2016;84(6):933-940. https://doi.org/10.1016/j.gie.2016.04.034 
63. Iglesias-Garcia J, Lindkvist B, Lariño-Noia J, AbdulkaderNallib I, Dominguez-Muñoz JE. Differential diagnosis of solid pancreatic masses: contrast-enhanced harmonic (CEHEUS), quantitative-elastography (QE-EUS), or both? United European Gastroenterol J. 2017;5(2):236-246. https://doi.org/10.1177/2050640616640635

64. Ignee A, Jenssen C, Arcidiacono PG, Hocke M, Möller K, Saftoiu A, Will U, Fusaroli P, Iglesias-Garcia J, Ponnudurai R, Petrone MC, Braden B, Burmester E, Dong Y, Atkinson NS, Dietrich CF. Endoscopic ultrasound elastography of small solid pancreatic lesions: a multicenter study. Endoscopy. 2018;50(11):1071-1079. https://doi.org/10.1055/a-0588-4941

65. Iordache S, Costache MI, Popescu CF, Streba CT, Cazacu S, Săftoiu A. Clinical impact of EUS elastography followed by contrast-enhanced EUS in patients with focal pancreatic masses and negative EUS-guided FNA. Med Ultrason. 2016;18(1):18-24. https://doi.org/10.11152/mu.2013.2066.181.ich

66. Carrara S, Auriemma F, Di Leo M, Rahal D, Preatoni P, Correale L, Anderloni A, Repici A. Endoscopic ultrasoundelastography (strain ratio) in the diagnosis of solid pancreatic lesions: A prospective cohort study. Endosc Ultrasound. 2017;6(Suppl 2):S54. https://doi.org/10.4103/2303-9027.218430

67. Hernández Mondragón OV, Velez Resendiz JM, Ruiz RR. Quantitative Elastography Versus Fine-needle Aspiration by Endoscopic Ultrasound for the Assessment of Pancreatic Solid Masses. J Clin Gastroenterol. 2019;53(7):e261-e268. https://doi.org/10.1097/MCG.0000000000001017

68. Ueno E, Umemoto T, Bando H, Tohno E, Waki K, Matsumura T. New quantitative method in breast elastography: fat-lesion ratio (FLR). En: Proceedings of the radiological society of North America scientific assembly and annual meeting. Chicago: Radiological Society of North America; 2007. p. 25-30.

69. Itoh Y, Itoh A, Kawashima H, Ohno E, Nakamura Y, Hiramatsu T, Sugimoto H, Sumi H, Hayashi D, Kuwahara T, Morishima T, Funasaka K, Nakamura M, Miyahara R, Ohmiya N, Katano Y, Ishigami M, Goto H, Hirooka Y. Quantitative analysis of diagnosing pancreatic fibrosis using EUS-elastography (comparison with surgical specimens). J Gastroenterol. 2014;49(7):1183-92. https://doi.org/10.1007/s00535-013-0880-4

70. Hirooka Y, Kuwahara T, Irisawa A, Itokawa F, Uchida H, Sasahira N, Kawada N, Itoh Y, Shiina T. JSUM ultrasound elastography practice guidelines: pancreas. J Med Ultrason (2001). 2015;42(2):151-74. https://doi.org/10.1007/s10396-014-0571-7

71. Pei Q, Zou X, Zhang X, Chen M, Guo Y, Luo H. Diagnostic value of EUS elastography in differentiation of benign and malignant solid pancreatic masses: a meta-analysis. Pancreatology. 2012;12(5):402-8. https://doi.org/10.1016/j.pan.2012.07.013

72. Mei M, Ni J, Liu D, Jin P, Sun L. EUS elastography for diagnosis of solid pancreatic masses: a meta-analysis. Gastrointest Endosc. 2013;77(4):578-89. https://doi.org/10.1016/j.gie.2012.09.035
73. Hu DM, Gong TT, Zhu Q. Endoscopic ultrasound elastography for differential diagnosis of pancreatic masses: a meta-analysis. Dig Dis Sci. 2013;58(4):1125-31. https://doi.org/10.1007/s10620-012-2428-5

74. Li X, Xu W, Shi J, Lin Y, Zeng X. Endoscopic ultrasound elastography for differentiating between pancreatic adenocarcinoma and inflammatory masses: a meta-analysis. World J Gastroenterol. 2013;19(37):6284-91. https://doi.org/10.3748/wjg.v19.i37.6284

75. Havre RF, Waage JR, Gilja OH, Ødegaard S, Nesje LB. Real-Time Elastography: Strain Ratio Measurements Are Influenced by the Position of the Reference Area. Ultraschall Med. 2012;33(6):559-568. https://doi.org/10.1055/s-0031-1273247

76. Zhang B, Zhu F, Li P, Yu S, Zhao Y, Li M. Endoscopic ultrasound elastography in the diagnosis of pancreatic masses: A meta-analysis. Pancreatology. 2018;18(7):833-840. https://doi.org/10.1016/j.pan.2018.07.008

77. Jafri M, Sachdev AH, Khanna L, Gress FG. The Role of Real Time Endoscopic Ultrasound Guided Elastography for Targeting EUS-FNA of Suspicious Pancreatic Masses: A Review of the Literature and A Single Center Experience. JOP. 2016;17(5):516-524.

78. Siddiqui AA, Brown LJ, Hong SK, Draganova-Tacheva RA, Korenblit J, Loren DE, Kowalski TE, Solomides C. Relationship of pancreatic mass size and diagnostic yield of endoscopic ultrasound-guided fine needle aspiration. Dig Dis Sci. 2011;56(11):3370-5. https://doi.org/10.1007/s10620-011-1782-z

79. Shiina T, Nightingale KR, Palmeri ML, Hall TJ, Bamber JC, Barr RG, Castera L, Choi BI, Chou YH, Cosgrove D, Dietrich CF, Ding H, Amy D, Farrokh A, Ferraioli G, Filice C, Friedrich-Rust M, Nakashima K, Schafer F, Sporea I, Suzuki S, Wilson S, Kudo M. WFUMB guidelines and recommendations for clinical use of ultrasound elastography: Part 1: basic principles and terminology. Ultrasound Med Biol. 2015;41(5):1126-47. https://doi.org/10.1016/j.ultrasmedbio.2015.03.009

80. Cosgrove D, Piscaglia F, Bamber J, Bojunga J, Correas JM, Gilja OH, Klauser AS, Sporea I, Calliada F, Cantisani V, D’Onofrio M, Drakonaki EE, Fink M, Friedrich-Rust M, Fromageau J, Havre RF, Jenssen C, Ohlinger R, Săftoiu A, Schaefer F, Dietrich CF; EFSUMB. EFSUMB guidelines and recommendations on the clinical use of ultrasound elastography. Part 2: Clinical applications. Ultraschall Med. 2013;34(3):238-53. https://doi.org/10.1055/s-0033-1335375

81. Hocke M, Braden B, Jenssen C, Dietrich CF. Present status and perspectives of endosonography 2017 in gastroenterology. Korean J Intern Med. 2018;33(1):36-63. https://doi.org/10.3904/kjim.2017.212

82. Altonbary AY, Hakim H, El-Shamy AM. Diagnostic Efficacy of Endoscopic Ultrasound Elastography in Differentiating Solid Pancreatic Lesions: A Single-Center Experience. Clin Endosc. 2019;52(4):360-364. https://doi.org/10.5946/ce.2018.160 